\title{
Tracing the behaviour of hexachlorobenzene in a paddy soil-rice system over a growth season
}

\author{
YANG Hua, ZHENG Minghui, ZHU Yongguan* \\ Research Center for Eco-Environmental Sciences, Chinese Academy of Sciences, Beijing 100085, China. E-mail: shufangyoung@ 163.com
}

Received for 21 March 2007; revised 22 April 2007; accepted 9 May 2007

\begin{abstract}
Hexachlorobenzene (HCB), a persistent organic pollutant (POP), has been found in paddy soils. To improve the understanding of HCB contamination in paddy soils, a laboratory simulative study was carried out to investigate the behavior of HCB in a paddy soil and rice plants. This study was divided into three experiments. First, an experiment aimed to examine the evaporation of HCB in paddy soil. In the second experiment, rice was planted in $10 \mathrm{mg} / \mathrm{kg} \mathrm{HCB}$ contaminated soil and after pot culture at 3, 6, 9, and 27 weeks (at maturity), both soil and plant samplings were scheduled to be sampled. The soil samples comprised rhizosphere soil, nonrhizosphere soil, and unplanted contaminated soil, whereas plant samples included shoots, roots, and rice grains (dehusked). Lastly, in this part, HCB in xylem saps was designed to be examined. The results showed that (1) the HCB translocation from paddy soil to rice by vaporization; (2) the HCB concentration in rice grains was surprisingly high; (3) the observed HCB decrease in rice rhizosphere offers a potential means for in situ HCB degradation; (4) HCB might not be transported along transpiration in rice.
\end{abstract}

Key words: HCB; paddy soil; rice; rhizosphere; xylem sap

\section{Introduction}

Hexachlorobenzene (HCB) is distributed widely throughout the environment because of its mobility and resistance to degradation (Jones, 2005). This compound moves through the environment presumably via a series of vaporization-deposition steps, that is, by a commonly called "grasshopper effect" (Wania and Mackay, 1995). As one of the persistent organic pollutants (POPs), HCB is listed high on the elimination table of the Stockholm Convention on Persistent Organic Pollutants (http://www.pops.int/documents/convtext/convtext_en.pdf), the priority list of persistent bioaccumulative toxic compounds by USEPA (http://www.epa.gov/pbt/hexa.htm), and a priority substance in the UN-ECE Convention on Long Range Transport of Air Pollutants (CLRTAP) protocol (Jones, 2005). HCB was first introduced in 1945 as a fungicide for the seeds of onions, sorghum, and other crops, and the agricultural use of HCB probably dominated HCB emissions in 1950s and 1960s (Bailey, 2001). Although the industrial HCB production has ceased in most countries by this time, it is still being generated inadvertently as a by-product and/or impurity in several chemical processes, such as the manufacturing of chlorinated solvents, chlorinated aromatics, and pesticides (Bailey, 2001). Existing field data indicate that soil is an important global reservoir for persistent organic pollutants

\footnotetext{
*Corresponding author. E-mail: ygzhu@ @rcees.ac.cn.
}

(Valle et al., 2005; Sweetman et al., 2005), and the variation of $\mathrm{HCB}$ concentration in soil with time mirrors its production levels (Barber et al., 2005). HCB in soil has received increasing attention (Zhang et al., 2006), although a majority of research center on the microbial transformation of HCB in soil (Matheus et al., 2000; Pavlostathis and Prytula, 2000; Adrian and Görisch, 2002; Brahushi et al., 2004; Tomoyas et al., 2007).

Contamination of plants, especially the food crops, which serve as the first link of the food-chain process, influences human health closely as they are more susceptible to contamination by pollutants in soil (Mclachlan, 1996; Su et al., 2005a; Su et al., 2005b; Su and Zhu, 2005, 2006). Thus, HCB contamination of crops deserves attention. There have been relevant reports on crops contaminated by HCB in soil (Nhan et al., 1999; Pastor et al., 2004; Darnerud et al., 2006).

Rice (Oryza sativa) is a staple crop in many Asian countries, particularly southern China. Rice is not only the main food for humans, but also an important feed for livestock. To the best of the authors' knowledge, there is little laboratory data examining the contamination of HCB in paddy soil and rice over a growth season. This information may improve the understanding of HCB contamination in paddy soils. From another standpoint, rice is grown typically in a wetland whose rhizosphere differs sharply from that of an upland. The rhizosphere is a zone of increased microbial density and activity at the root/surface (Curl and Truelove, 1986). Plant and microorganisms 
often have symbiotic relationships making the root zone or rhizosphere an area of very active microbial activity (Siciliano and Germida, 1998). It is therefore of interest to explore whether the rhizosphere of rice has a potential for remediating HCB, because almost all earlier studies on HCB phytoremediation were conducted with upland plants such as carrots (Tao et al., 2005), maizes (Wild et al., 2005), and grasses (Wilson and Mehary, 2003).

In this research, a study on the HCB behavior in a paddy soil-rice system was initiated to investigate (1) the volatile levels of HCB in a paddy soil and the potential contamination to rice seedlings; (2) the accumulation of $\mathrm{HCB}$ in different tissues of $O$. sativa as the plants grow in a given system; (3) the rhizospheric effect of $O$. sativa on HCB degradation in a paddy soil; (4) HCB transportation along transpiration stream.

\section{Materials and methods}

\subsection{Soil and plant preparation}

Paddy soil $(0-30 \mathrm{~cm})$ was collected from Jiaxing, Zhejiang Province, China. The characteristics of the soil are as follows: soil organic matter (SOM) of $2.8 \%, \mathrm{pH} 6.1$, the field water-holding capacity of $40 \%$, and the CEC (cation exchange capacity) of $17.94 \mathrm{cmol} / \mathrm{kg}$. The background level of HCB in this soil was below the detection limit (the analytical method followed is mentioned later). A total of $20-\mathrm{kg}$ soil was sieved through a $2-\mathrm{mm}$ sieve. HCB $(0.2 \mathrm{~g})$ was dissolved in $150-\mathrm{ml} n$-hexane and 250 $\mathrm{g}$ of this soil was soaked in the solvent, followed by a vigorous stirring until the solvent was evaporated so that the soil could be evenly spiked. This contaminated soil was mixed thoroughly with the uncontaminated soil. Then, the soil was aged for about one month, maintained with $50 \%$ water-holding capacity (based on dry soil). The soil was sieved again together with base fertilizer $(0.247$ $\mathrm{g} \mathrm{K}_{2} \mathrm{SO}_{4} / \mathrm{kg}$ soil, $0.428 \mathrm{~g} \mathrm{CO}\left(\mathrm{NH}_{2}\right)_{2} / \mathrm{kg}$ soil, $0.323 \mathrm{~g}$ $\mathrm{CaHPO}_{4} \cdot 2 \mathrm{H}_{2} \mathrm{O} / \mathrm{kg}$ soil) before being packed into plastic cylindrical pots $(9.5 \mathrm{~cm}$ i.d. $\times 14 \mathrm{~cm}$ in height, lined with plastic bags, roughly 600-g soil in each pot). To ease the sampling of the rhizosphere soil, root bags were used to confine the extension of the seedling roots. A root bag (4.1 cm i.d. and $14 \mathrm{~cm}$ in height) made of 360-mesh nylon cloth was filled with the spiked soil and placed along the geometrical central axial inside a pot.

The preparation of uncontaminated soil (the control soil) was the same as the contaminated one described in the previous paragraph but without the HCB addition procedure.

Seeds of Jiahua-1 rice cultivar were used in this study. Rice seeds were disinfected in $10 \%$ hydrogen peroxide solution for about $10 \mathrm{~min}$, germinated in perlite medium, and placed in a controlled growth chamber (14-h photoperiod, $260-350 \mu \mathrm{E} /\left(\mathrm{m}^{2} \cdot \mathrm{s}\right)$ active radiation, temperature regulated to $25^{\circ} \mathrm{C}$ during day and $20^{\circ} \mathrm{C}$ at night, and with $70 \%$ relative humidity). One month later, the uniform seedlings were transferred into soil (one plant per pot) or nutrient solution (six plants per pot) and were kept in the growth chamber. Irrigation methods in the experiment followed the farming practices but using deionized water, that is, flush irrigation at the tiller stage, light irrigation at the end of tiller stage, and wetting alternating with drying when the frutification occurred.

\subsection{Experimental design}

The overall work was divided into three sections. The first two sections were intended for two main objectives and the third one was a supplement. The three experiments were conducted successively.

\subsubsection{Experiment 1}

One month after germination, four uniform rice seedlings were transferred into four PVC cylindric pots (7.5 cm i.d. $\times 14 \mathrm{~cm}$ height) packed with uncontaminated soil. Each pot was covered with a circular PVC lid having a hole on the lid to allow for seedling growth. The edge of the hole was lined with sponge to avoid damage of the seedlings. The lid was halved along the diameter for watering (flush irrigation) convenience. The seams between two cuts and between the edges of holes and shoots were all sealed with polytetrafluoroethylene tape. The four unvegetated pots (flush irrigation), without lids, containing artificially contaminated soil were interspersed among the seedling pots. All pots were arranged along a circumference and placed in the same growth chamber as described later (Fig.1). When watering, the pots with seedlings were removed from the chamber to avoid any possible HCB contamination to soil. One month after being transferred into the pots, plant samples were sampled for analysis of HCB contents in shoots.

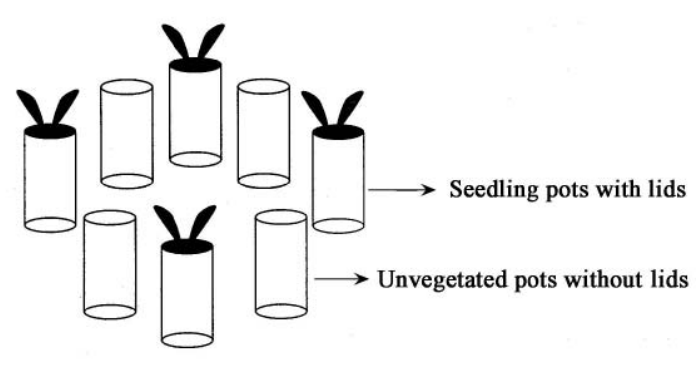

Fig. 1 Schematic set-ups for Experiment 1.

\subsubsection{Experiment 2}

Two treatments were set up in this experiment: the unvegetated contaminated soil (the control soil) and the vegetated contaminated soil. Each treatment was prepared in quadruplicate. The sampling periods were as follows: the first sampling was after the seedlings were cultivated for three weeks in the pots, with subsequent twice samplings every three weeks thereafter until the plants bore grains (about 27 weeks in this experiment) for the last sampling. For sampling, the soil samples (the control soil, nonrhizosphere soil, and rhizosphere soil) and the plant samples (roots and shoots) were collected. 


\subsubsection{Experiment 3}

Hydroponic culture was carried out to examine HCB in xylem saps. After one month of germination, six seedlings per pot (PVC cylindric pots, $10.5 \mathrm{~cm}$ i.d. $\times$ $15 \mathrm{~cm}$ height) as one replicate were cultured in Kimura B nutrient solution (the components of solution followed Zhu et al., 2004). The seedlings were grown for up to two months before initiating treatment. Two treatments were applied: contaminated nutrient solution that were saturated by superfluous crystalline HCB and uncontaminated nutrient solution without HCB. Seedlings were fed in these solutions for $3 \mathrm{~d}$. Each treatment was prepared in quadruplicate. Xylem saps were collected after $3 \mathrm{~d}$.

\subsection{Sampling}

\subsubsection{Experiments 1 and 2}

The rhizosphere soil from the root bags was sampled with a stainless steel spoon; the soil adhering to the roots was carefully scrapped off by avoiding sampling the surface/bottom soil. Between the pot walls and root bag walls and away from the surface/bottom soil, the nonrhizosphere soil was sampled. Similarly, avoiding the surface/bottom soil and those adjacent to pot walls, the control soil was sampled. As to the plant samples, they were carefully removed from the soil after the rhizosphere soil was sampled, rinsed with the tap water, then with the deionized water, and cut with stainless steel scissors to separate the tissues: roots, shoots, and grains. Furthermore, the grains were dehusked and ground with a mortar and pestle for subsequent analyses. All samples were immediately frozen at $-20^{\circ} \mathrm{C}$ for further freeze-drying.

\subsubsection{Experiment 3: xylem sap collection}

To collect xylem sap, plants were decapped $3 \mathrm{~cm}$ above the pot and the cut surface was washed with distilled water. After blotting excess moisture, the stubble was closely fit in an aluminium foil sleeve wrapped by a rubber band. The sap was collected with a microliter syringe for $3 \mathrm{~h}$ between 18:00-21:00 (Shen and Wang, 2002). The roots were saved to extract HCB when xylem sap collection was finished and the extraction procedure was similar to Experiment 1.

\subsection{Extraction, cleanup, and analysis}

Soil samples were freeze-dried, ground with a mortar and pestle, and then sieved to $0.6 \mathrm{~mm}$. Plant samples were freeze-dried, ground with a mortar and pestle with the aid of liquid nitrogen. All samples were extracted in Soxhlet apparatus (as a cleaning step, the Soxhlet apparatus had been pre-extracted for about $8 \mathrm{~h}$ with the $3-\mathrm{g}$ silica gel in each thimble using 250-ml $n$-hexane) with $250-\mathrm{ml} n$ hexane for $18 \mathrm{~h}$ after being soaked in $n$-hexane for about $6 \mathrm{~h}$. Into each Soxhlet cellulose thimble, the following substances were weighed and put sequentially: $3 \mathrm{~g}$ silica gel, $10 \mathrm{~g}$ soil sample or plant sample mixed with $20 \mathrm{~g}$ anhydrous sodium sulfate, and $10 \mathrm{~g}$ anhydrous sodium sulfate.

The sample extract was transferred to a 500-ml separatory funnel equipped with a Teflon stopcock, shaken with 15 $\mathrm{ml}$ concentrated sulfuric acid, and washed with deionized water until $\mathrm{pH}$ value of discarded water layer was equal to 7. The extract was further dried with anhydrous sodium sulfate, concentrated with a rotary evaporator, and finally evaporated to $1 \mathrm{ml}$ for the soil sample extract and $0.5 \mathrm{ml}$ for the plant sample extract in a Kuderna-Danish (K-D) concentrator under a gentle nitrogen stream.

Liquid-liquid extraction was used to extract HCB in the sap. At a time, 5-ml $n$-hexane was added into the sap and the liquid mixture was shaken manually. Supernatants were combined after three extractions and concentrated to $100 \mu \mathrm{l}$ for gas chromatograph (GC) detection.

Samples were analyzed using Agilent GC 6820 equipped with ${ }^{63} \mathrm{Ni}$ electron capture detector (ECD), DB1 column $(15 \mathrm{~m} \times 0.53 \mathrm{~mm}$ i.d., $0.32-\mu \mathrm{m}$ film thickness $)$, in a splitless mode. The initial oven temperature was set at $120^{\circ} \mathrm{C}$, held for $3 \mathrm{~min}$, ramped at $10^{\circ} \mathrm{C} / \mathrm{min}$ to $150^{\circ} \mathrm{C}$, maintained for $2 \mathrm{~min}$, and then raised to the final temperature of $200^{\circ} \mathrm{C}$ at the rate of $15^{\circ} \mathrm{C} / \mathrm{min}$. Both injector and detector temperature were at $250^{\circ} \mathrm{C}$. The carrier gas was nitrogen at $1.0 \mathrm{ml} / \mathrm{min}$. The working standard solutions were prepared with crystalline HCB and $n$-hexane; the $R^{2}$ of the external standard calibration curves is $>0.99$.

The recoveries were $102 \%, 98.5 \%, 115 \%, 98 \%$, and $65 \%$, for the soil, root, shoot, rice-husk, and rice-grain, respectively.

\subsection{Reagents and glasswares}

Crystalline HCB was purchased from the Beijing Chemical Reagent Co. $n$-Hexane (A.R.) was obtained from the Tianjin Fuchen Chemical Reagent Factory. Silica gel (100200 mesh) was provided by the Qingdao North District Haihua Desiccant Factory and all other inorganic reagents were all analytical grade and supplied by the Beijing Chemical Reagent Co. The deionized water was prepared using ORGANO (Organo Co. Ltd., Japan). Anhydrous sodium sulfate and silica gel were heated at $660^{\circ} \mathrm{C}$ and $550^{\circ} \mathrm{C}$ for $6 \mathrm{~h}$, respectively, in a furnace and then kept in the desiccator before use.

All glassware except for Soxhlet apparatus (which was pre-extracted as described earlier) were rinsed with $n$-hexane thrice, cleaned in an ultrasonic cleaner with detergent, and dried in an oven, except that the K-D concentrators were air-dried.

\subsection{Statistical analysis}

Significance of the data was evaluated using SPSS version 11.5 for Windows with a general linear model for analysis of variance and least significant difference (LSD) with $p \leqslant 0.05$ for comparing differences of HCB concentrations in samples.

\section{Results and discussion}

\subsection{Experiment 1}

The HCB concentration in shoots was (on dry weight basis) $0.085 \pm 0.006 \mathrm{mg} / \mathrm{kg}$ (mean $\pm \mathrm{SE}, n=4$ ), indicating that the shoots of rice plants in uncontaminated soil were contaminated by volatile HCB from the contaminated soil. 
This observation confirms the highly volatile nature of HCB and supports the conclusion by Mclachlan (1996) and Yao et al. (2006) that the considerably higher HCB fugacity in soil than in air makes the soil a source of HCB content in the atmosphere. This result might also be useful in assessing human exposure to contaminated wetland.

\subsection{Experiment 2}

The HCB recoveries from the control, rhizosphere, and nonrhizosphere soils showed a general decrease of $81.2 \%$ and $54.5 \%$, respectively, with the sampling time (Fig.2). In the first three samplings, the differences of HCB recoveries among the control soil, rhizosphere soil, and nonrhizosphere soil were not significant $(p \leqslant 0.05)$. It seemed that the rhizosphere did not enhance the HCB degradation over the first 9 weeks. However, it is noteworthy that the initial HCB recoveries in rhizosphere soil became perceivably higher, although slightly, than those in nonrhizosphere and the control soil. In contrast to the first three samplings, the significant difference between rhizosphere soil and the control/nonrhizosphere soil occurred at the fourth sampling time, that is, after 27 weeks of the cultivation, in which the HCB recovery from the rhizosphere soil dropped to nearly half of that from the nonrhizosphere soil.

The combined results from Experiments 1 and 2 indicate that, other than the degradation factor, the declining trend of HCB levels in soil may partly result from the volatilization loss. Another probable reason for the decrease is the aging effect that makes a compound in soil become more resistant to extraction with increasing contact time. It is thus appropriate to use mild, selective extractants to assess the bioavailability of POPs (Kelsey et al., 1997; Dean et al., 2004). As $n$-hexane used in this experiment is thought to be a mild solvent, the extraction results probably demonstrate the HCB bioavailability in soil (Tao et al., 2004). Thus this decreasing trend may also reveal the decreasing bioavailability of HCB in soil with increasing aging time. The bioavailability may be responsible for the result of slightly higher $\mathrm{HCB}$ recoveries in rhizosphere soil than that in nonrhizosphere soil in the early stage. This higher bioavailability might be related to the release of low molecular weight organic acids, such as citric and malic acids from roots, and as the soil matrix might be impaired

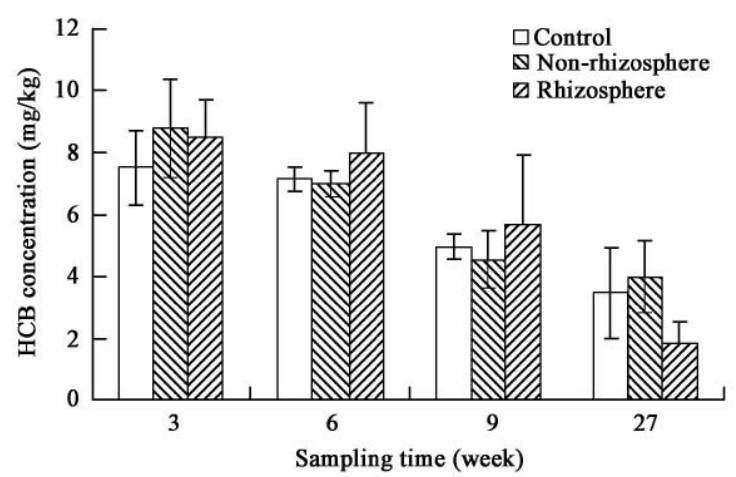

Fig. 2 HCB concentrations (on dry weight basis) in control, rhizosphere, nonrhizosphere soils versus time in Experiment 2. Sampling times were $3,6,9$, and 27 weeks after the rice seedlings were cultivated in the pots. Data represent the mean $\pm \operatorname{SE}(n=4)$. by the organic acids, the sequestered organic pollutants is released (Mattina et al., 2003). Similar hypotheses have been assumed for the bioavailability of PCDD/PCDF (polychlorinated dibenzo- $p$-dioxin/polychlorinated dibenzofuran) (Hülster et al., 1994).

Rhizospheric influence became well noted with the presence of roots: the bigger the roots grow, the more apparent was the rhizoshperic effect. On the other hand, the bioavailability of $\mathrm{HCB}$ in rhizosphere may increase with time as discussed earlier. These might explain the significant difference between the HCB concentrations in rhizosphere soil and nonrhizosphere soil noted in the fourth sampling. Considering that HCB is extremely resistant to degradation, more time is needed for its phytoremediation than other organic pollutants. The intriguing result provides motivation to further investigate whether wetland plant that possesses aerenchyma capable of releasing oxygen to its local environment could be a better candidate for HCB phytoremediation as, until now, there are few upland plants that are reported to remediate HCB effectively in soil. This idea has already been taken into account in the constructed wetland for wastewater treatment (Bezaruah and Zhang, 2004a, b), and a further experiment testing this thought is underway in this laboratory.

In this study, HCB concentrations in roots were almost always the highest compared to those with other tissues in each sampling. With plant growth, an approximately constant concentration of HCB in roots followed a sudden steep decline of $65.3 \%$ (Fig.3), even as HCB concentration in shoots decreased gradually. With regard to the total $\mathrm{HCB}$, the amounts in roots were appreciably higher than that in shoots (Table 1). It was unexpected that the HCB concentration in rice grains is considerably higher than in husks, which is contrary to the previous finding on PCB (polychlorinated benzene) (Bi et al., 2002).

It was not surprising to find that the highest $\mathrm{HCB}$ concentration in plants was in roots, because roots were in direct contact with the soil and thus had more access to HCB. There are two possible pathways of the HCB translocation into the roots: the direct diffusion of gaseous HCB into the roots and the uptake of dissolved HCB from soil solution into roots (Ecker and Horak, 1994). The growth of roots may dilute the HCB concentrations,

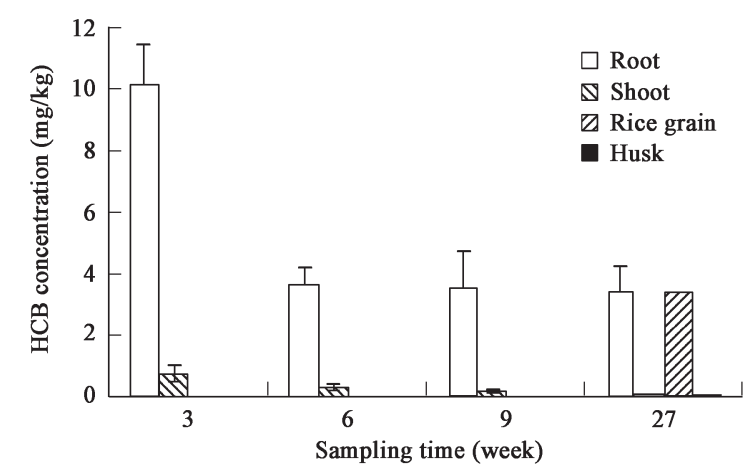

Fig. 3 HCB concentrations (on dry weight basis) in roots, shoots, rice grains, and husks versus time in Experiment 2. Sampling times were 3, 6,9 and 27 weeks after cultivation in the pots. Data represent the mean \pm SE $(n=4)$. 
Table 1 Total amount of HCB and BCFs with roots and shoots

\begin{tabular}{llllll}
\hline Sampling time & & 3 weeks & 6 weeks & 9 weeks & 27 weeks \\
\hline Total amount of HCB $(\mu \mathrm{g})$ & Roots & $1.03 \pm 0.28$ & $3.4 \pm 0.78$ & $9.87 \pm 4.67$ & $17.57 \pm 6.52$ \\
& Shoots & $0.48 \pm 0.13$ & $0.71 \pm 0.13$ & $1.00 \pm 0.27$ & $0.58 \pm 0.064$ \\
BCFs* & Roots & $1.21 \pm 0.25$ & $0.46 \pm 0.03$ & $0.73 \pm 0.46$ & $2.19 \pm 1.27$ \\
& Shoots & $0.09 \pm 0.03$ & $0.04 \pm 0.01$ & $0.03 \pm 0.01$ & $0.04 \pm 0.02$ \\
\hline
\end{tabular}

*BCFs are defined as the HCB concentration in plant parts divided by that in rhizosphere soil. Data represent the mean \pm SE $(n=4)$.

even though the total amount of $\mathrm{HCB}$ in roots appeared to increase with the expansion of root surface areas. As to the shoot contamination, there are three possible pathways: the root uptake and subsequent translocation into shoots via the transpiration process, the direct vapor uptake via the atmosphere, and the deposition of contaminated soils and dusts on plant cuticles and the subsequent contaminant diffusion through plant surfaces. Although the Experiment 3 failed to prove the first possibility, it cannot be ruled out because HCB translocation within pumpkin plants has been observed (Ecker and Horak, 1994). In Experiment 1, the second possibility has been confirmed, indicating that HCB evaporated from soil was taken up by the leaves. Exotic compounds may penetrate into leaves in two waysthrough the stomata or through the epidermis. For gases and vapors, the primary mode of penetration is through stomata; for example, the experiments with hypostomatous plant leaves revealed that benzene vapor is taken up mainly through stomata (Ugrekhelidze et al., 1997). The natural protective covering of higher plant leaves consists of nonliving cuticle and its waxy coating. Leaf waxes act as a barrier to the penetration of organic pollutants and with the aging of leaves, the quantity of epicuticular waxes accumulated on the leaf surface increases (Nakajima et al., 1996). This probably leads to the more intensive uptake of hydrocarbons by young leaves than by old ones (Harvey et al., 2002). Besides, the same dilution effect for HCB in roots because of plant growth applies to the HCB concentration in shoots.

The very high HCB concentration in rice grains is surprising although a contamination during the sampling processes was inevitable. Meanwhile, rather low HCB contents in shoots were detected (Table 1). This maybe ascribed in part to the lower lipid content in shoots than in grains, in light of the fact that plant tissues rich in lipid contents may contain correspondingly higher levels of organic contaminants (Li et al., 2005). However, further studies are needed to substantiate this point.

In this study, it is emphasized that as roots directly contact the rhizosphere soil rather than bulk soil, the bioconcentration factor (BCF) (Table 1) was defined as the HCB concentration in plant divided by that in the rhizosphere soil. In the first three samplings, the BCFs of HCB with roots fluctuated around 1, which is similar to the findings with the roots of the monocotyledoneae (maize, oat, barley): the BCF was nearly 1 at the initial growth period (Schroll and Scherunert, 1992). Factors such as lipid content and surface area may lead to the change of BCFs (Jiao et al., 2006), and further study is required to investigate the pending question. In contrast to roots, the BCFs for shoots were far less than 1 in all samplings.

\subsection{Experiment 3}

For each pot, ca. 500- $\mu$ l sap was pooled from six individual plants, and no HCB was detected by GC, irrespective of $\mathrm{HCB}$ addition to the nutrient solution. The concentration in roots was $0.826 \pm 0.004 \mathrm{mg} / \mathrm{kg}$ (mean $\pm \mathrm{SE}, n=4)$. This experiment was meant to examine if $\mathrm{HCB}$ can be transported from roots to shoots along the transpiration stream. The results here showed that under the experimental conditions described for this study, translocation of HCB to above ground may be negligible. It might be difficult for HCB to permeate cortex cells and further distribution of $\mathrm{HCB}$ between plant components and water should be examined.

\section{Conclusions}

To summarize, it was demonstrated that HCB could evaporate from a paddy soil to contaminate a wetland crop such as rice. A significant decreasing HCB concentration trend $(p \leqslant 0.05)$ with contact time in a paddy soil can be observed. This trend may stem from aging, a process that could reduce HCB bioavailability in soil, and then decreased HCB concentration that could be extracted, as well as from an abiotic and biotic degradation process. The observed low HCB concentration in rhizosphere soil after a long contact time makes the rice plant a potential species for HCB phytoremediation despite the decontamination process being slow. The active water transportation in rice might fail to explain the fact that $\mathrm{HCB}$ was detected in the shoots. The high HCB concentration in rice grains may be an indication and a consequence of the enhanced HCB accumulation into a high-lipid plant component.

\section{Acknowledgements}

This work was supported by the Ministry of Science and Technology (No. 2007CB407304). The authors would like to thank all colleagues for their valuable and positive inputs to this work. Thanks are also in order to those who reviewed the manuscript.

\section{References}

Adrian L, Görisch H, 2002. Microbial transformation of chlorinated benzenes under anaerobic conditions. Research in Microbiology, 153: $131-137$.

Bailey R E, 2001. Global hexachlorobenzene emissions. Chemosphere, 43: $167-182$.

Barber L J, Sweetman J A, Wijk D, Jones K C, 2005. Hexachlorobenzene in the global environment: Emissions, levels, distribution, trends and processes. Science of the Total Environment, 349: 1-44.

Bezaruah A N, Zhang T C, 2004a. pH, redox, and oxygen microprofiles in rhizosphere of bulrush (Scirpus validus) in a constructed wetland treating municipal wastewater. Biotechnol Bioeng, 88: 60-70. 
Bezbarush A N, Zhang T C, 2004b. Quantification of oxygen release by bulrush (Scirpus validus) roots in a constructed treatment wetland. Biotchnol Bioeng, 89: 309-318.

Bi X H, Chu S G, Meng Q Y, Xu X B, 2002. Movement and retention of polychlorinated biphenyls in a paddy field of WenTai area in China. Agriculture, Ecosystems and Environment, 89(3): 241-252.

Brahushi F, Dörfler U, Schroll R, Munch J C, 2004. Stimulation of reductive dechlorination of hexachlorobenzene in soil by inducing the native microbial activity. Chemosphere, 55: 1477-1484.

Curl E A, Truelove B, 1986. The Rhizosphere. Berlin, Germany: Springer.

Darnerud P O, Atuma S, Aune M, Bjerseliu R, Glynn A, Petersson G K, Becker W, 2006. Dietary intake estimations of organohalogen contaminants (dioxin, PCB, PBDE and chlorinated pesticides, e.g. DDT) based on Swedish market basket data. Food and Chemical Toxicology, 44: 1597-1606.

Dean J R, Scott W C, 2004. Recent developments in assessing the bioavailability of persistent organic pollutants in the environment. Trends in Analytical Chemistry, 23: 609-618.

Ecker S, Horak O, 1994. Pathways of HCB-contamination to oil pumpkin seeds. Chemosphere, 29: 2135-2145.

Harvey P J, Campanella B F, Castro P M L, Harms H, Lichtfouse E, Schaffner A R, Smrcek S, Daniele W R, 2002. Phytoremediation of polyaromatic hydrocarbons, anilines, phenols. Environ Sci and Pollu Res, 9: 29-47.

Hülster A, Müller J F, Marschner H, 1994. Soil-plant transfer of polychlorinated dibenzo- $p$-dioxins and dibenzofurans to vegetables of the cucumber family (cucurbitaceae). Environ Sci Technol, 28: 11101115

Jiao X C, Xu F L, Dawson R, Chen S H, Tao S, 2007. Adsorption and absorption of polycyclic aromatic hydrocarbons to rice roots. Envrion Pollut, 148(1): 230-235.

Jones K, 2005. Hexachlorobenzene-sources, environmental fate and risk characterization. European Chlorinated Solvent Association (Euro Chlor).

Kelsey J W, Kottler B D, Alexander M, 1997. Selective chemical extractants to predict bioavailability of soil-aged organic chemicals. Environ Sci Technol, 31: 214-217.

Li H, Sheng G Y, Chiou C T, Xu O, 2005. Relation of organic contaminant equilibrium sorption and kinetic uptake in plants. Environ Sci Technol, 39: 4864-4870.

Matheus D R, Bononi V L R, Machado K M G, 2000. Biodegradation of hexachlorobenzene by basidiomycetes in soil contaminated with industrial residues. World Journal of Microbiology $\mathcal{E}$ Biotechnology, 16: 415-442.

Mattina M I, Lannucci-Berger W, Musante C, White J C, 2003. Concurrent plant uptake of heavy metals and persistent organic pollutants from soil. Environmental Pollution, 124: 375-378.

Mclachlan M S, 1996. Bioaccumulation of hydrophobic chemicals in agicultural food chains. Environ Sci Technol, 30: 252-259.

Nakajima D, Kojima E, Iwaya S, Suzuki J, Suzuki S, 1996. Presence of 1-hydroxypyrene conjugates in woody plant leaves and seasonal changes in their concentrations. Environ Sci Technol, 30: 16751679 .

Nhan D D, Am N M, Carvalho F P, Villeneuve J P, Cattini C, 1999. Organochlorine pesticides and PCBs along the coast of north Vietnam. The Science of the Total Environment, 237/238: 363-371.

Pastor D, Sanpera C, González-Solís J, Ruiz X, Albaigés J, 2004. Factors affecting the organochlorine pollutant load in biota of a rice field ecosystem (Ebro Delta, NE Spain). Chemosphere, 55: 567-576.

Pavlostathis S G, Prytula M T, 2000. Kinetics of the sequential microbial reductive dechlorination of hexachlorobenzene. Environ Sci Technol, 34: 4001-4009.
Schroll R, Scheunert I, 1992. A laboratory system to determine separately the uptake of organic chemicals from soil by plant roots and by leaves after vaporization. Chemosphere, 24: 97-108.

Shen B, Wang X, 2002. Physiological activities of root system in two inter-subspecific hybrid rice combinations. Chinese J of Rice Sci, 16(2): 146-150.

Siciliano S D, Germida J J, 1998. Bacterial inoculants of forage grasses enhance degradation of 2-chlorobenzoic acid in soil. Environ Toxicol Chem, 16: 1098-1104.

$\mathrm{Su}$ Y H, Zhu Y G, 2005. Influence of lead on atrazine uptake by rice (Oryza sativa L.) seedlings from nutrient solution. Environ Sci $\mathcal{E}$ Pollut Res, 12(1): 21-27.

Su Y H, Zhu Y G, Du X, 2005a. Co-uptake of atrazine and mercury by rice seedlings from water. Pesticide Biochemistry and Physiology, 82: 226-232.

Su Y H, Zhu Y G, Lin A J, Zhang X H, 2005b. Interaction between cadmium and atrazine during uptake by rice seedlings (Oryza sativa L.). Chemosphere, 60: 802-809.

Su Y H, Zhu Y G, 2006. Bioconcentration of atrazine and chlorophenols into roots and shoots of rice seedlings. Environmental Pollution, 139: 32-39.

Sweetman A J, Valle M D, Prevedouros K, Jones K C, 2005. The role of soil organic carbon in the global cycling of persistent organic pollutants (POPs): interpreting and modeling field data. Chemosphere, 60(7): 959-972.

Tao S, Guo L Q, Wang X J, 2004. Use of sequential ASE extraction to evaluate the bioavailability of DDT and its metabolites to wheat roots. I: soils with various organic carbon contents. The Science of the Total Environment, 320: 1-91.

Tao S, Xu F L, Wang X J, Liu W X, Gong Z M, Feng J Y et al., 2005. Organochlorine pesticides in agricultural soil and vegetables from Tianjin, China. Environ Sci Technol, 39: 2494-2499.

Tomoyasu H, Takako I, Kokyo O, Ryuichi S, 2007. Biodegradation of chlordane and hexachlorobenzenes in river sediment. Chemosphere, 67: 428-434.

Ugrekhelidze D, Kort F, Kvesitadze G, 1997. Uptake and transformation of benzene and toluene by plant leaves. Ecotoxicology and Environmental Safety, 37: 24-29.

Valle M D, Jurado E, Dachs J, Sweetman A J, Jones K C, 2005. The maximum reservoir capacity of soils for persistent organic pollutants: implications for global cycling. Environmental Pollution, 134(1): 153-164.

Wania F, Mackay D, 1995. A global distribution model for perisistent orgainic chemicals. Sci Total Environ, 160/161: 211-232.

Wild E, Dent J, Thomas G O, Jones K C, 2005. Direct observation of organic contaminat uptake, storage, and metabolism within plant roots. Environ Sci Technol, 39: 3695-3702.

Wilson S C, Meharg A A, 2003. Investigation of organic xenobiotic transfers, partitioning and processing in air-soil-plant systems using microcosm apparatus. Part II: comparing the fate of chlorobenzenes in grass planted soil. Chemosphere, 53: 583-591.

Yao Y, Tuduri L, Harner T, Blanchard P, Waite D, Poissant L et al., 2006. Spatial and temporal distribution of pesticide air concentrations in Canadian agricultural regions. Atmospheric Environment, 40: 4339-4351.

Zhang H B, Luo Y M, Zhao Q G, Wang M H, Zhang G L, 2006. Residues of organochlorine pesticides in Hong Kong soils. Chemosphere, 63: 633-641.

Zhu X J, Yang J S, Liang Y C, Lou Y S, Yang X L, 2004. Effects of exogenous calcium on photosynthesis and its related physiological characteristics of rice seedlings under salt stress. Scientia Agricultura Sinica, 37(10): 1497-1503. 